This is the accepted version of the following article:

Hayati P., Sori B., Rezvani A.R., Morsali A., Gutierrez A.. Sonochemical synthesis and characterization of a novel hetro-binuclear metal organic nano polymer based on picolinic acid ligand. J ournal of Molecular Structure, (2017). 1150. : 404 - . 10.1016/j. molstruc. 2017.09.006,

which has been published in final form at https://dx.doi.org/10.1016/j.molstruc.2017.09.006 @ https://dx.doi.org/10.1016/j.molstruc. 2017.09.006. This manuscript version is made available under the CC-BY-NC-ND 4.0 license http://creativecommons.org/licenses/by-nc-nd/4.0/ 


\section{Sonochemical synthesis and characterization of a novel hetro-binuclear metal organic nano polymer based on picolinic acid ligand}

Payam Hayati ${ }^{\text {ac }}$ BagherSouria Ali Reza Rezvani ${ }^{\mathrm{a}}$ Ali Morsali ${ }^{\mathrm{b}}$ AngelGutierrez ${ }^{\mathrm{d}}$

a Department of Chemistry, Faculty of Sciences, University of Sistan and Baluchestan, P.O. Box 98135-674, Zahedan, Iran.

b Department of Chemistry, Faculty of Sciences, Tarbiat Modares University, P.O. Box 14115-4838, Tehran, Islamic Republic of Iran.

c Catalan Institute of Nanoscience and Nanotechnology (ICN2), CSIC and the Barcelona Institute of Science and Technology, Campus UAB, Bellaterra, 08193 Barcelona, Spain.

d Departamento de Química Inorgánica I Facultad de Ciencias Químicas Universidad Complutense, 28040 Madrid, Spain.

Abstract

Nanoparticles of one new lead and $K$ coordination polymer (CP), $\{[\mathrm{Pb} 6(\mathrm{pyc}) 6(\mathrm{~N} 3) 7 \mathrm{~K}] .1 / 2 \mathrm{H} 2 \mathrm{O}\} \mathrm{n}(1) \mathrm{Hpyc}=$ picolinic acid ligand, has been synthesized by use of a sonochemical process and characterized by scanning electron microscopy (SEM), X-ray powder diffraction (XRPD), Fourier transform infrared spectroscopy (FTIR) spectroscopy and elemental analyses. The single crystal X-ray data of compound 1 imply that the $\mathrm{Pb}$ ion is seven coordinated. The thermal stability of compound 1 has been studied by thermogravimetric (TG) and differential scanning calorimetry (DSC). The role of temperature, reaction time and ultrasound irradiation power on the size and morphfology of the nano-structured compound obtained from 1, have been investigated. Results indicate that an increase of temperature and sonication power and a decrease in time reaction led to a decrease of particle size. 


\section{Introduction}

An enormous amount of attention in recent years has been accorded to crystal engineering and the construction of coordination polymers with structural topologies because of their potential application in several fields such as optoelectronics, separation science, gas storage, catalysis, adsorption, host-guest chemistry, electro functional materials, luminescence, non-linear optics and magnetism [1], [2], [3], [4], [5]. A large amount of coordination polymers with a wide variety of structural motifs has been prepared through the variation of reagents and reaction conditions [6], [7], [8]. The structure and the properties of such materials rely on several factors, including the oxidation state and coordination geometry of central metals, structural chemistry of organic spacers, nature of solvents, $\mathrm{pH}$ value, temperature, the counteranion and molar ratio of central metal to organic ligand. Moreover, weak interactions (H-bonding, $\Pi-\pi$ stacking, van der Waals) could also play a significant role in ultimate control the network [9], [10], [11], [12], [13]. Lead ion has a larger radius, variable stereochemical activity and flexible coordination environment, so this metal ion provides an exceptional opportunity for new metal coordination compounds [14], [15], [16], [17], [18], [19], [20]. The bridging modes of azido ligand (N3) are shown in

Scheme 1 These features besides the presence of co-ligands, have resulted in several polynuclears coordination compounds forming 1D, 2D and 3D architectures with interesting polymeric topologies [21], [22], [23], [24], [25], [26], [27], [28], [29].

Cavitation mechanism generated in the irrigant solution adjacent to a tooth surface can be used for cleaning [30], [31]. This suggestion was reinforced by the detection by Lea et al. of transient cavitation generated through the vibratory motion of the scaler tips [32]. Cavitation occurs in liquids once the acoustic pressure during the rarefaction phase of an oscillation becomes more negative compared to saturated 
vapor pressure of the liquid, resulting in the forming of cavities or bubbles. Cavitation results in both chemical and mechanical effects. The former includes the formation of excited states with consequent light emission and reactive free radical species from pyrolysis of the liquid and any dissolved species. Types of mechanical effects are acoustic streaming and the formation of microjets that effect on solid surface adjacent to collapsing cavities [33], [34], [35], [36], [37], [38], [39], [40].

Several filler for polymeric nanocomposite and the effects of ultrasound radiation on chemical reactions were reported in the recent works [44], [45], [46], [47], [48], [49], [50], [51], [52], [53]. In this manuscript, we have designed a simple sonochemical process to make nano structures of $\left\{[\mathrm{Pb} 6(\mathrm{pyc}) 6(\mathrm{~N} 3) 7 \mathrm{~K}] \cdot \frac{1}{2} \mathrm{H} 2 \mathrm{O}\right\} \mathrm{n}$, The ultrasonic power, sonicating time, the temperature of reaction were the parameters that have been changed for reaching the optimized condition. Among the potential characterization methods such as scanning electron microscopy (SEM) and powder X-ray diffraction (PXRD) were used.

\section{Experimental}

\subsection{Materials and physical techniques}

Starting reagents for the synthesis were purchased and used without any purification from industrial suppliers (Sigma-Aldrich, Merck and others). Elemental analyses (carbon, hydrogen, and nitrogen) were performed employing a Heraeus Analytical Jena, Multi EA 3100 CHNO rapid analyzer. Fourier transform infrared spectra were recorded on a FT-IR JASCO 680-PLUS spectrometer as KBr pellets in the 4000-400 cm-1 spectral range. Thermogravimetric analysis (TGA) and differential scanning 
calorimetry (DSC) of the title compound were performed on a computer-controlled STA - PT 1500 apparatus. Single-phased powder sample of 1 - 1 and crystal structure compound 1 was loaded into alumina pans and heated with a ramp rate of $10{ }^{\circ} \mathrm{C} / \mathrm{min}$ from room temperature to $600{ }^{\circ} \mathrm{C}$ under argon atmosphere. X-ray diffraction experiment was carried out at the MoKa wavelength at ambient temperature. A micro focused Rigaku mm003 source with integrated confocal caxFlux double bounce optic was employed, and Bragg peak measurement was performed by an HPAD Pilatus $200 \mathrm{~K}$ detector. The crystal data were collected in the "CAI de Difracción de Rayos X, UCM". A colourless crystal was resin epoxy coated and mounted on a Bruker Smart CCD diffractometer using graphite monochromated Mo-Ka radiation $(\lambda=0.71073 \AA$ ) operating at $50 \mathrm{kV}$ and $25 \mathrm{~mA}$. Data were collected over a reciprocal space hemisphere by combination of three exposure sets. Each frame exposure time was $20 \mathrm{~s}$ covering $0.3^{\circ}$ in $\omega$. The cell parameters were determined and refined by least-squares fit of all reflections collected. The first 50 frames were recollected at the end of the data collection to monitor crystal decay, and no appreciable decay was observed. The structure was solved by direct methods and refined by applying full-matrix least-squares on F2 with anisotropic thermal parameters for the non-hydrogen atoms. The hydrogen atoms were included with fixed isotropic contributions at their calculated positions determined by molecular geometry. All the calculations were carried out with SHELX97, using the OLEX2 software package [35], [36], [37]. A multi wave ultrasonic generator (Sonicator_3000; Misonix, Inc. Farmingdale, NY, USA), equipped with a converter/transducer and titanium oscillator (horn), $12.5 \mathrm{~mm}$ in diameter, operating at $20 \mathrm{kHz}$ with a maximum power output of $600 \mathrm{~W}$, were used for the ultrasonic irradiation. Melting points were measured on an electrothermal 9100 apparatus and 
are uncorrected. X-ray powder diffraction (XRD) measurements were performed using an X'pert diffractometer manufactured by Philips with monochromatized Cuka radiation and simulated XRD powder patterns based on single crystal data were prepared using the Mercury software [38]. The samples were characterized with a scanning electron microscope (SEM) (Philips XL 30 and S-4160).

\subsection{Synthesis of $\left\{[\mathrm{Pb} 6(\mathrm{pyc}) 6(\mathrm{~N} 3) 7 \mathrm{~K}] \cdot \frac{1}{1} / \mathrm{H} 2 \mathrm{O}\right\} \mathrm{n}$ (1) as single crystal}

Lead(II) nitrate $(1 \mathrm{mmol}, 0.331 \mathrm{~g})$, picolinic acid ( $1 \mathrm{mmol}, 0.123 \mathrm{~g}$ ), potassium chloride ( $1 \mathrm{mmol}, 0.074 \mathrm{~g})$ and sodium azide $(2 \mathrm{mmol}, 0.130 \mathrm{~g})$ were loaded into one arm of a branch tube and both of the arms were filled slowly by water. The chemical bearing arm was immersed in an oil bath kept at $60^{\circ} \mathrm{C}$. Crystals were formed on the inside surface of the arm kept at ambient temperature, After 5 days, colorless crystals were deposited in the cooler arm were filtered off, washed with water and air dried. White crystals, m.p $>300{ }^{\circ} \mathrm{C}$. Anal. Calc. for C72H48K2N54O27Pb12: C: 18.53, H: 1.02, O: 9.26\%; Found C: 18.47, H: 0.96, O: 9.21\%. IR (selected bands for compound 1; in cm-1): 3320(w), 2036(s), 1609(w), 1562(w), 1363(s), 699(s)cm-1.

\subsection{Synthesis of $\{[\mathrm{Pb} 6(\mathrm{pyc}) 6(\mathrm{~N} 3) 7 \mathrm{~K}] .1 / 2 \mathrm{H} 2 \mathrm{O}\} \mathrm{n}$ (1) nano-structures under ultrasonic irradiation}

Lead(II) nitrate high-density ultrasonic probe immersed directly into the solution of Lead(II) nitrate $(10 \mathrm{ml}, 0.1 \mathrm{M})$ in water, then into this solution, a proper volume of picolinic acid ligand, potassium chloride and sodium azide in water solvent $(10 \mathrm{ml}$, $0.1 \mathrm{M})$ was added in a drop wise manner. . In order to study the effect of time, 
temperature and power on size and morphology of nano-structured compound 1, the ultrasonic irradiation was performed for different times (30 min, $1 \mathrm{~h}$ ), at different temperature $\left(50^{\circ} \mathrm{C}, 70^{\circ} \mathrm{C}\right)$ and with different power $(0 \mathrm{~W}, 60 \mathrm{~W})$. The obtained precipitates were filtered, subsequently washed with water and then dried. Yield $0.315 \mathrm{~g}$ (76.82\% based on final product). Product 1 (nano structure):

The concentration was maintained constant in all syntheses. The obtained precipitates were filtered, washed with water and dried in air. m.p $>300{ }^{\circ} \mathrm{C}$. Anal. Calc. for C72H48K2N54O27Pb12: C: 18.53, H: 1.02, O: 9.26\%; Found C: 18.50, H: 1.01, O: $9.18 \%$. IR (selected bands for compound 1; in cm-1): 3326(w), 2020(s), 1609(w), 1564(w), 1362(s), 698(s)cm-1.

\section{Results and discussion}

The compound of formula $\left\{[\mathrm{Pb} 6(\mathrm{pyc}) 6(\mathrm{~N} 3) 7 \mathrm{~K}] \cdot \frac{1}{2} \mathrm{H} 2 \mathrm{O}\right\} \mathrm{n} \quad$ (1) $\quad(\mathrm{pyc}=2$ picolinate, $\left.\mathrm{C} 6 \mathrm{H} 4 \mathrm{NO}^{-}-\right)$crystallizes in the space group. The potassium atom and one azido anion lie on a three-fold axis, while the rest of the atoms surround them. In this way the asymmetric unit is built up by one lead atom, one picolinate and one azido anions, along with the corresponding fractions of potassium and azide found on special positions.

The six lead atoms in the molecular unit are symmetry equivalents and are located in the corners of an almost regular octahedron. The inner part of this octahedron is occupied by the azido anion that lies on the threefold axis and that is coordinated to the six lead atoms through its terminal nitrogen atoms. 
Every lead atom is seven coordinated to four nitrogen atoms and three oxygen ones (Fig. 1). All the picolinate groups are bridging two adjacent lead atoms in a double chelating way. The picolinate is bonded through the pyridine nitrogen and one carboxylate oxygen atom to the first lead while it is also chelating the second lead atom through the bidentate carboxylate group. The bond distances between 2.41 and $2.78 \AA$ are in the range typical for $\mathrm{PbO}$ and $\mathrm{PbN}$ bonds and angle between $\mathrm{PbOPb}$ and $\mathrm{PbNPb}$ are $145.88^{\circ}$ and $109.94^{\circ}$, respectively. The other $\mathrm{PbN}$ distances correspond to bonds between three azido groups and the lead atom.

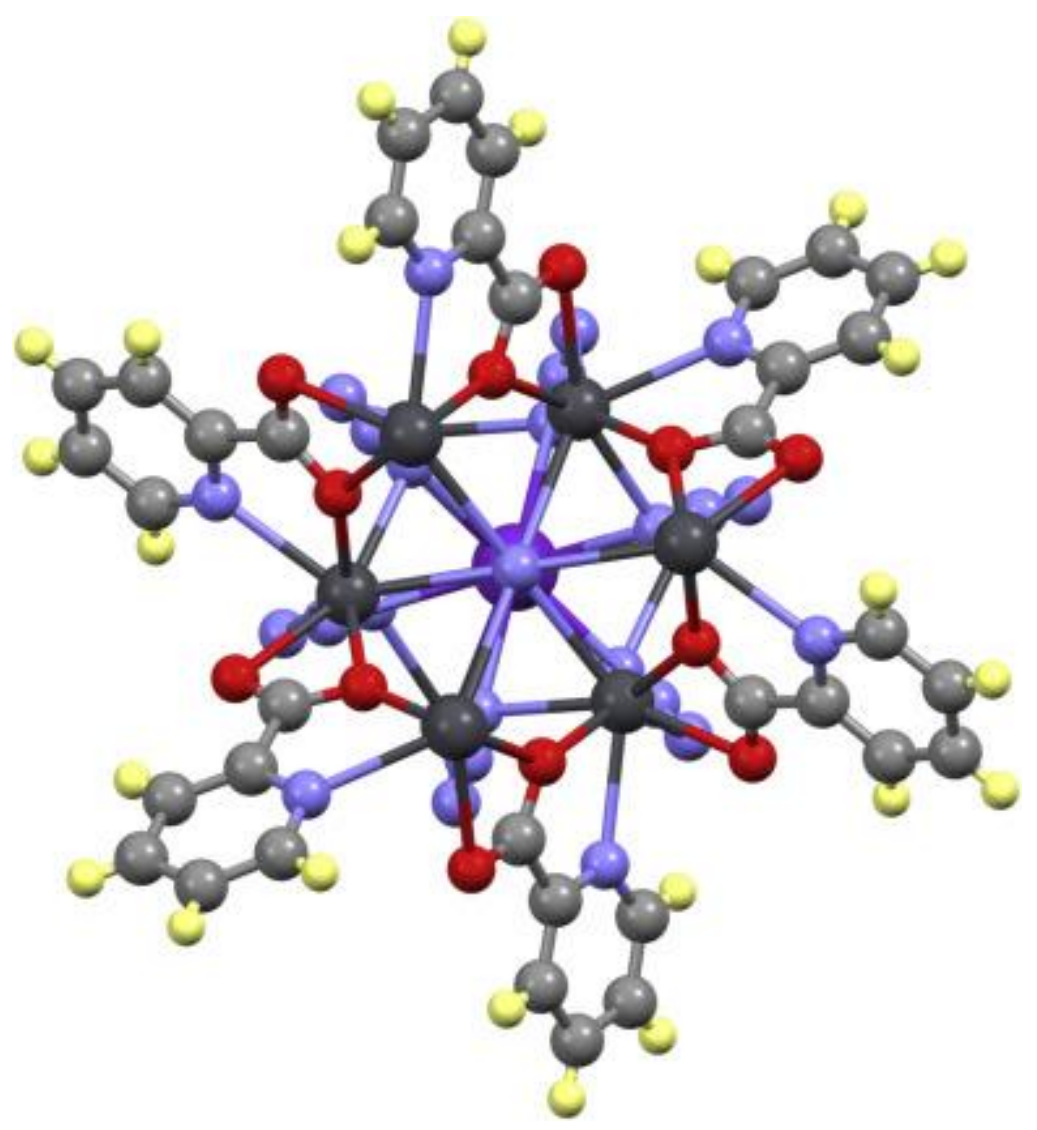

Fig. 1. View along the $c$ axis showing the coordination of the lead atoms (dark grey). 
Between two lead layers is located the potassium ion surrounded by six azido groups in an octahedral environment (Fig. 2). The KN bond distance is $2.817(18) \AA$ and angle between PbNK is $83.92^{\circ}$. Each of these azido anions is also coordinated to one lead atom through the same terminal nitrogen at a distance of 2.595(13) $\AA$ and it is also bridging through the opposite nitrogen atom to one lead of the second layer, with a bond distance of $2.884(15) \AA$. These bridges extend the interaction along the $\mathrm{c}$ axis, giving rise to the formation of a one-dimensional coordination polymer, by alternation of layers of lead-picolinate and potassium-azido groups (Fig. S1).

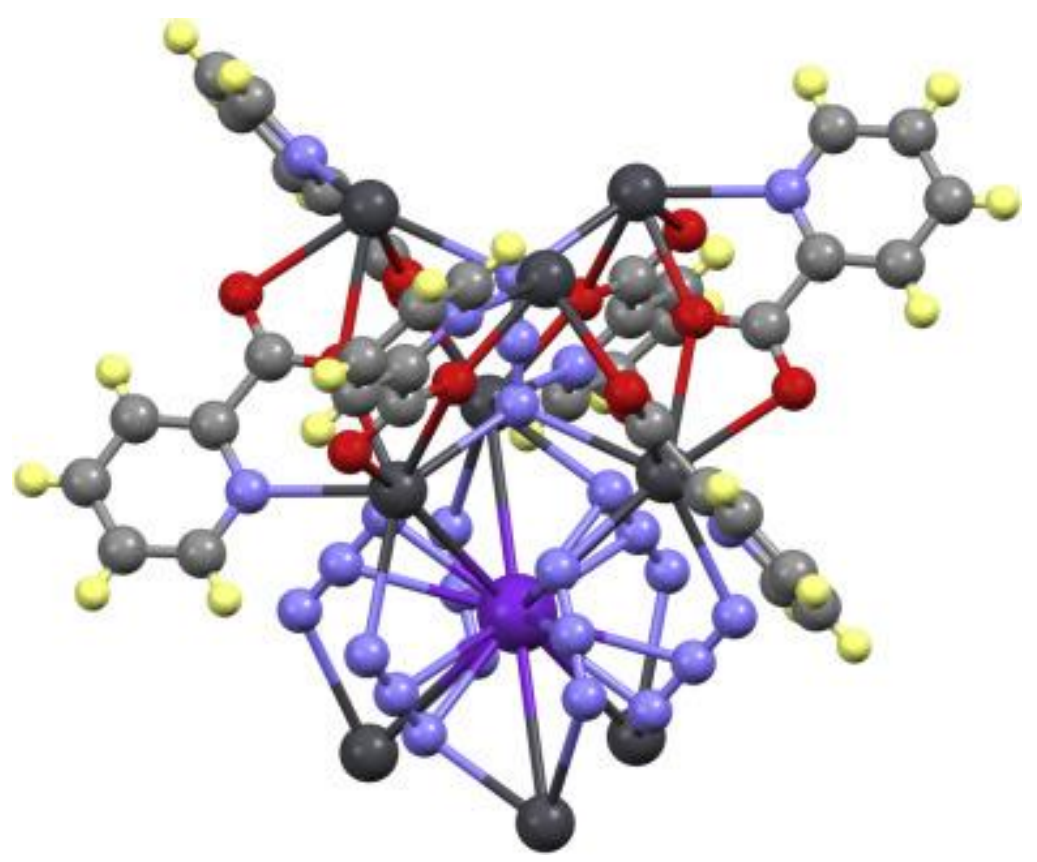

Fig. 2. View of the molecular unit showing the coordination sphere of the potassium ion (violet) surrounded by six azido anions. (For interpretation of the references to colour in this figure legend, the reader is referred to the web version of this article.) 
The chains are arranged parallel in the crystal, leaving channels that hold the disordered solvent water molecules (Fig. 3). Every chain is surrounded by six other same chains forming van-der-waals bonded hexagonal packing of cylinders in 1 (Fig.S2).

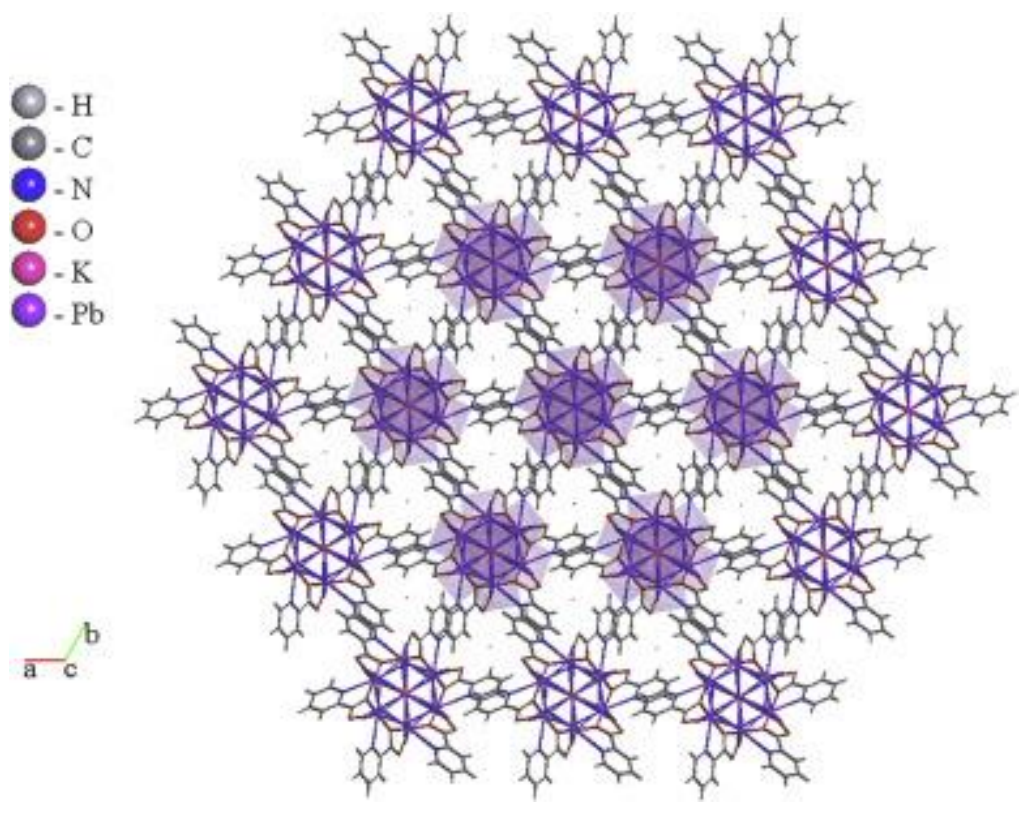

Fig. 3. Packing of the polymeric chains showing the channels with the disordered solvent water molecules.

Single crystal X-ray diffraction analysis (Table 1, Table 2) of compounds 1 was carried out. In summary, 1 units forming respective CPs of lead and potassium consisting of 1D chains running along c-axis ([001] direction) (Fig. S1). Within the stacks the picolinate linkers are perpendicular each other and separated by a distance of $9.930 \AA$ in 1 (these values are between the gravity centers of neighboring pyridine rings within the stacks). These distances are too long for the effective $\pi-\pi$ interactions between the aromatic rings within the stacks (Fig. S3). Also, azide bridges are coordinated like $\mu 1,1^{\prime}, 2,3$ while covered the $K$ atom as cage. Such azide 
bridges sound really special while this type of azide bridge coordination has not been reported yet, therefore kind of topology this compound is unknown (Fig. 1 and Scheme 1).

Table 1. Crystal data and structures refinement $[\mathrm{Pb}(\mathrm{L}) \mathrm{K}(\mathrm{N} 3) 2] \mathrm{n}(1)$.

\begin{tabular}{|c|c|}
\hline Empirical formula & $0.33\left(\mathrm{C}_{36} \mathrm{H}_{24} \mathrm{KN}_{2 \gamma} \mathrm{O}_{12} \mathrm{~Pb}_{\mathrm{b}}\right) \cdot 0.5(\mathrm{O})$ \\
\hline Formula weigh & 777.69 \\
\hline Temperature & $296(2) K$ \\
\hline Wavelength & $0.71073 \AA$ \\
\hline Crystal system & Trigonal \\
\hline Space group & 13 \\
\hline Unit cell dimensions & $\begin{array}{l}a-12.8106(7) \hat{A} \alpha-90.00^{\circ} \\
b-12.8106(7) \hat{A}, \beta-90.00^{\circ} \\
c-9.9298(8) \hat{A}, \gamma-120.00^{\circ}\end{array}$ \\
\hline Volume & $1411.27(19) \hat{A}^{3}$ \\
\hline Z, Z & 3,0 \\
\hline Density(calculated) & $2.745 \mathrm{~g} / \mathrm{cm}^{3}$ \\
\hline Absorption coefficient & $17.99 \mathrm{Mg} / \mathrm{m}^{3}$ \\
\hline$F(000)$ & 1048 \\
\hline Crystal size & $0.18 \times 0.13 \times 0.12 \mathrm{~mm}^{3}$ \\
\hline Theta range for data collection & $1.8-28.5^{\circ}$ \\
\hline н & $17.99 \mathrm{~mm}^{-1}$ \\
\hline Index ranges & $\begin{array}{l}16<h<16 \\
12<\mathrm{k}<16 \\
13<\mathrm{l}<13\end{array}$ \\
\hline$(\sin \theta / A) \max$ & $0.671 \Lambda^{-1}$ \\
\hline Theta(max) & $28.5^{\circ}$ \\
\hline Radiation type & Mo $\mathrm{K} \alpha$ \\
\hline Refinement method & Full- matrix least-squares on $P^{2}$ \\
\hline Goodness- of- fit on $\mathrm{F}^{2}$ & 1.062 \\
\hline Refinement & $\begin{array}{l}\mathrm{R}\left[\mathrm{F}^{2}>2 \sigma\left(\mathrm{F}^{2}\right)\right]-0.039 \\
\mathrm{wR}\left(\mathrm{F}^{2}\right)-0.114\end{array}$ \\
\hline Kint & $\underset{\text { aoss }}{S}-1.06$ \\
\hline Largest diff. peak and hole & $2.68, \quad 0.099 \mathrm{eA}^{-3}$ \\
\hline CCDC no. & 1511740 \\
\hline
\end{tabular}

Table 2. Selected bond lengths/Å for compound $\{[\mathrm{Pb} 6(\mathrm{pyc}) 6(\mathrm{~N} 3) 7 \mathrm{~K}] .1 / 2 \mathrm{H} 2 \mathrm{O}\} \mathrm{n}$.

\begin{tabular}{llll}
\hline $\mathrm{K}(1)-\mathrm{N}(2)^{\mathrm{iv}}$ & $2.817(18)$ & $\mathrm{N}(2)-\mathrm{Pb}(1)$ & $2.595(13)$ \\
$\mathrm{K}(1)-\mathrm{N}(2)^{\mathrm{v}}$ & $2.817(18)$ & $\mathrm{O}(1)-\mathrm{Pb}(1)$ & $2.415(7)$ \\
$\mathrm{K}(1)-\mathrm{N}(2)^{\mathrm{vi}}$ & $2.817(18)$ & $\mathrm{O}(2)-\mathrm{Pb}(1)^{\mathrm{i}}$ & $2.644(9)$ \\
$\mathrm{K}(1)-\mathrm{N}(2)^{\mathrm{vii}}$ & $2.817(18)$ & $\mathrm{Pb}(1)-\mathrm{O}(2)^{\mathrm{ii}}$ & $2.644(9)$ \\
$\mathrm{K}(1)-\mathrm{N}(2)^{\mathrm{v}}$ & $2.817(18)$ & $\mathrm{Pb}(1)-\mathrm{N}(5)$ & $2.827(5)$ \\
$\mathrm{K}(1)-\mathrm{N}(3)^{\text {viii }}$ & $3.140(12)$ & $\mathrm{Pb}(1)-\mathrm{K}(1)$ & $3.9242(5)$ \\
$\mathrm{K}(1)-\mathrm{N}(3)^{\mathrm{v}}$ & $3.140(12)$ & $\mathrm{N}(1)-\mathrm{Pb}(1)$ & $2.567(10)$ \\
$\mathrm{K}(1)-\mathrm{N}(3)^{\mathrm{vii}}$ & $3.140(12)$ & $\mathrm{N}(6)-\mathrm{N}(5)^{\mathrm{mi}}$ & $1.172(14)$ \\
$\mathrm{K}(1)-\mathrm{N}(3)^{\mathrm{jiv}}$ & $3.140(12)$ & $\mathrm{K}(1)-\mathrm{N}(2)^{\mathrm{iv}}$ & $2.817(18)$ \\
$\mathrm{K}(1)-\mathrm{N}(3)^{\mathrm{vi}}$ & $3.140(12$ & $\mathrm{K}(1)-\mathrm{N}(2)^{\mathrm{v}}$ & $2.817(18)$ \\
\hline
\end{tabular}

Symmetry transformations used to generate equivalent atoms. (i) $1+y, 1-x+y, 1-z$; (ii) $x-y$, $-1+x, 1-z$; (iii) $2-x,-y, 1-z$; (iv) $1+y, 1-x+y,-z$; (v) 2-x + y, 1-x, z; (vi) 1-y, -1+x-y, z; (vii) $x-y$, $-1+x,-z ;$ (viii) $2-x,-y,-z$. 
<smiles>[Y17]NN([M])N([M])[M]</smiles>

$\mu-1,1^{\prime}, 2,3$<smiles>CNN</smiles><smiles>[M]N[M]</smiles>

$\mu-1,1$

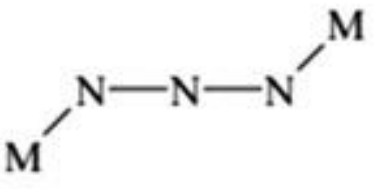

$\mu-1,3$

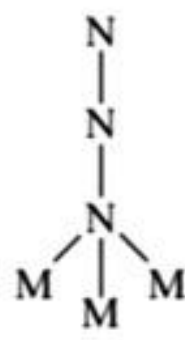

$\mu-1.1 .1$

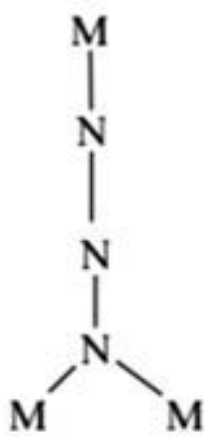

$\mu-1,1,3$

Scheme 1. Common bridging modes of the azido ligand.

Bravais Friedel Donnay Harker (BFDH) analysis was carried out in order to estimate the faces that are supposed to appear in the crystals morphology. This analysis considers the effect of symmetry operations on the interplanar distances of crystal faces [41]. Predicted crystal morphologies of complex 1 are shown in (Fig. S4). In almost all cases there is a good match between the predicted and observed morphology. It should be noted that in the cases of 1 , the growth of the coordination material takes place along the [010] directions. Also, Nanocrystals of compound 1 was obtained in aqueous solution by ultrasonic irradiation.

The IR spectra show characteristic absorption bands for picolinic acid ligand in compound 1. The IR spectrum of compound 1 show the characteristic stretching frequency of $\mathrm{OH}$ group observed at about $3442 \mathrm{~cm}-1$ the relatively broad absorption bands, The absorption bands with variable intensity in the frequency range 1700$1750 \mathrm{~cm}-1$ correspond to CO stretching frequency from the carbonyl of the picolinic acid ligands. Also, characteristic band of the CO stretching frequency carboxylic acid group appears at 1000-1300 cm-1. The absorption bands around 2141 and 2109 $\mathrm{cm}-1$ related to stretching frequency of the $\mathrm{N} 3$ group. Also, aromatic $\mathrm{CH}$ stretching 
frequency appears at around $3035 \mathrm{~cm}-1$. The elemental analysis and IR spectra of the nano-structure produced by the sonochemical method as well as the bulk material produced by the branched tube method are indistinguishable (Fig.S5).

Fig. 4 shows the XRD patterns of compounds 1 simulated from single crystal X-ray data. While the experimental XRD patterns of compounds 1 prepared by the sonochemical process is shown in Fig. 4. For 1 compound, acceptable matches, with slight differences in $2 \theta$, were observed between the simulated and experimental powder X-ray diffraction patterns. This indicates that the compound 1 obtained by the sonochemical process as nano-structures is identical to that obtained by single crystal diffraction. The significant broadening of the peaks indicates that the particles are of nanometer dimensions. Fig. 4 shows if the pick is sharper, then crystal lattice will be more regular. Also, for crystal and nano structures compound 1 is concluded which have same phase. This indicates that the compound 1 obtained son chemically is essentially identical to that obtained by conventional single crystal evaporation. Estimated from the Sherrer formula for the calculation of particle sizes from the broadening of the XRD peaks $D=0.891 N / \beta \cos \theta$, where $D$ is the average grain size, $\lambda$ is the $X$-ray wavelength $(0.15405 \mathrm{~nm})$, and $\theta$ and $\beta$ are the diffraction angle and full-width at half maximum of an observed peak, respectively, the average size of the particles was found to be around $50-165 \mathrm{~nm}$ for compound 1 which is in agreement with the value obtained from the SEM image (Fig. 4, Fig. 6). 


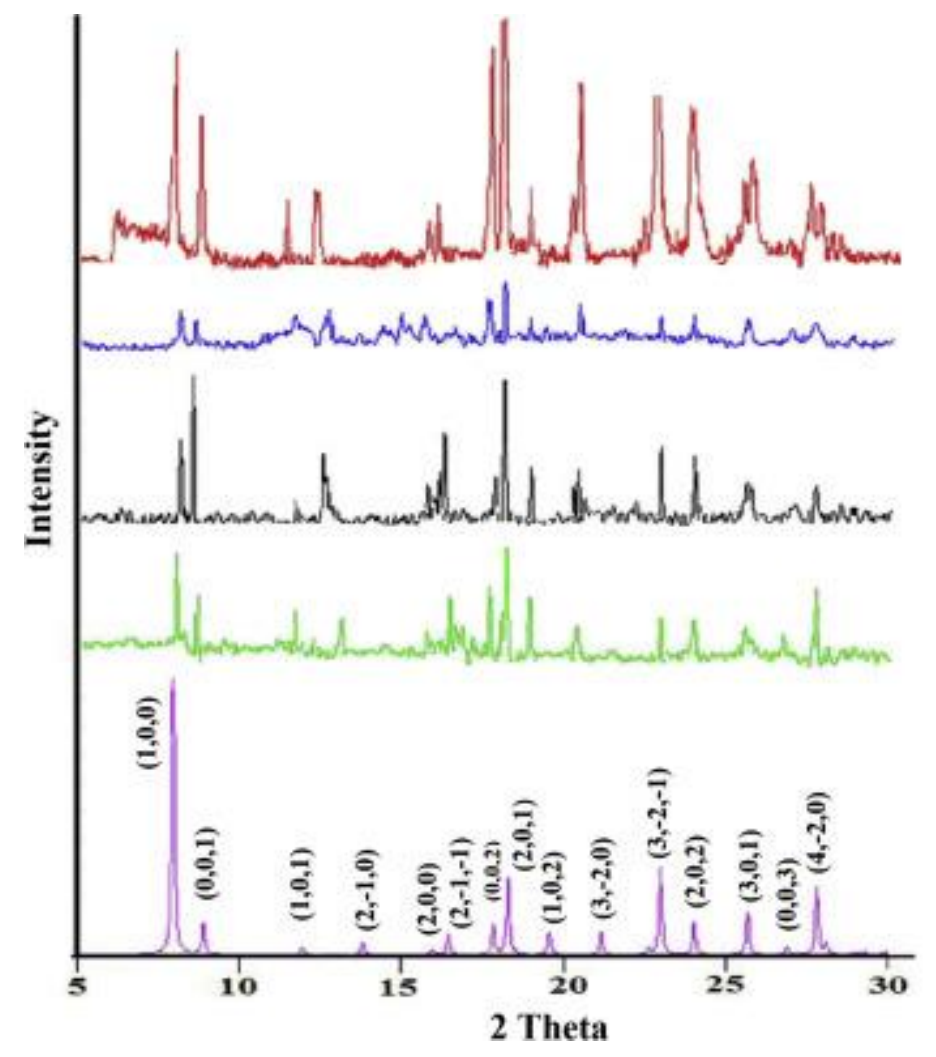

Fig. 4. (a) PXRD patterns corresponding to the simulation of compound 1 and the micro/nano-structured systems (b) 1-1,(c) 1-2, (d)1-3, (e) 1-4.

In order to look at the thermal stability of the four compounds, thermogravimetric (TG), differential scanning calorimetry (DSC) were carried out for compounds 1 between 30 and $600{ }^{\circ} \mathrm{C}$ under nitrogen flow.

The TG curve of compound 1 indicates that the compound does melt and is stable up to $100^{\circ} \mathrm{C}$ at that temperature it begins to decompose (Fig. 5). A first weight loss over $110-120{ }^{\circ} \mathrm{C}$ corresponding to the loss of coordinated and lattice water molecules with one endothermic peak. Pyridine aromatic ring and one carboxylate groups of ligand carry out in range of $320-460{ }^{\circ} \mathrm{C}$, respectively. Decomposition of the aromatic rings of pyridine aromatic ring and carboxylate groups of picolinate ligand takes place in two steps with two endothermic between from 328 to $347^{\circ} \mathrm{C}$ 
and $405-461{ }^{\circ} \mathrm{C}$, respectively. Mass loss calculations show that the final decomposition product is PbKN3 (Fig. 5).

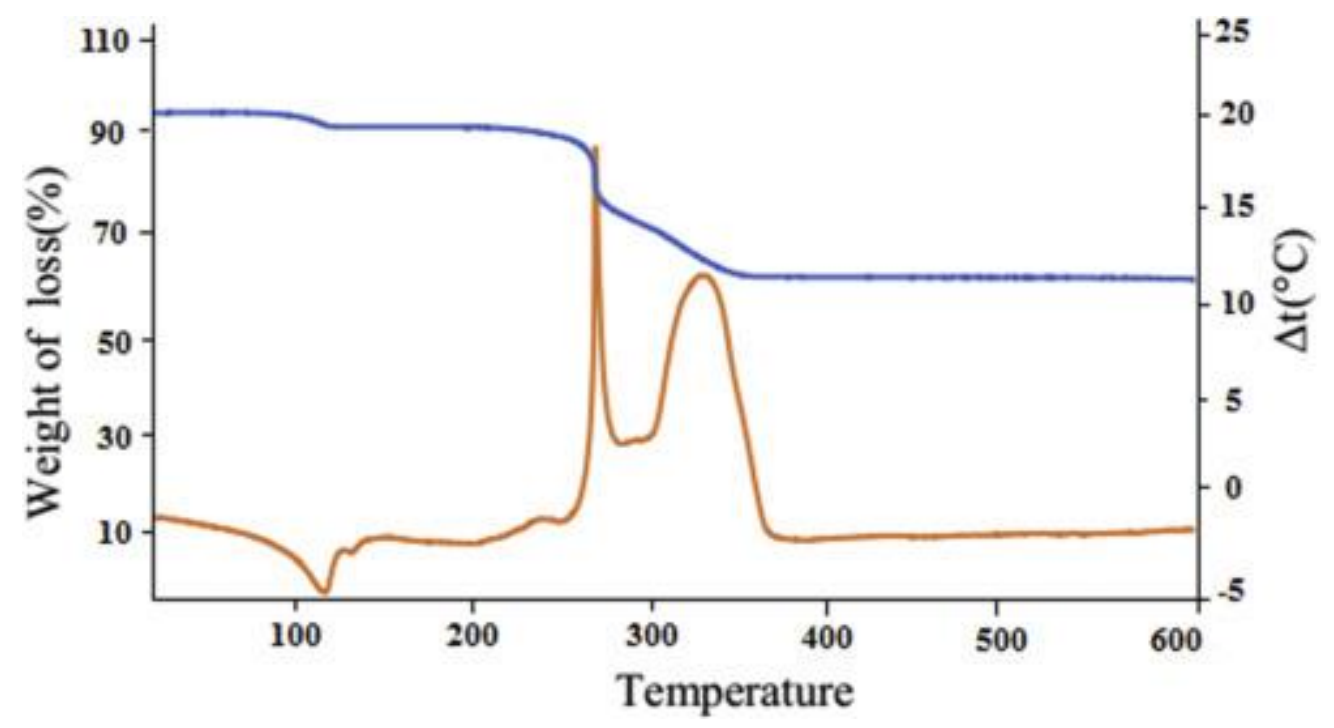

Fig. 5. Thermal behavior of compound 1 .

The whole process of ultrasound waves are based on acoustic cavitation through three steps of the formation, growth and collapse of bubbles inside a liquid exposed to ultrasound. Collapsing bubbles possess high temperature $(5000 \mathrm{~K})$ and high pressure (500 atm). In this method the reaction takes place in three different regions including inner environment of the cavitation bubbles, interfacial region and the bulk solution according to the nature of solvent, reactants as well as their vapor pressure [42]. The unstable high-temperature and high-pressure field create a proper conditions for the anisotropic growth of nanocrystals. On the other hands, we believe that ultrasound is responsible for a higher surface area and smaller particle size of the products. Extreme conditions during cavitation bubble collapse initiate both physical and chemical processes. Due to implosive collapse of bubbles, a shock 
wave generates which propagates out into the liquid medium. Bubble collapse near a solid surface disorders the spherical symmetry of the bubble and microjets form subsequently. These physical progressions make ultrasonic irradiation a highly effective and suitable means to mix liquids, erode solid surfaces, and facilitate interparticle collisions. Shock waves can accelerate suspended solid particles in the liquid. Interparticle collisions can reach velocities of hundreds of metres per second, causing alterations in particle size distributions, particle morphologies, and surface compositions. However, particle fragmentation is observed. Finally, the interparticle collisions result in induce striking changes in surface morphology, composition, and reactivity [43].

Various conditions for preparation of compounds 1 nano-structure were summarized in Table 3. In this table, sample 1-1, were studied without power ultrasound and the other samples were studied under variable temperature, time and power ultrasound. In order to research the role of power ultrasound irradiation on the character of product, reactions were performed under completely different power ultrasound irradiation. Results show a decrease in the particles size as increasing power ultrasound irradiation [39], [40].

Table 3. The influence of temperature, reaction time and sonication power on the size of compound 1 particle.

\begin{tabular}{|c|c|c|c|c|}
\hline Compound 1 samples & $\mathrm{T}\left({ }^{\circ} \mathrm{C}\right)^{4}$ & $\mathrm{~T}(\min )^{b}$ & Sonication (input power) (W) & SEM $^{\mathrm{C}}$ \\
\hline $1-1$ & 50 & 60 & 0 & 168 \\
\hline $1-2$ & 50 & 60 & 60 & 87 \\
\hline $1-3$ & 50 & 30 & 60 & 67 \\
\hline $1-4$ & 70 & 60 & 60 & 52 \\
\hline
\end{tabular}

a Reaction temperature. b Reaction time. c Average diameter (nm). 
In sample 1 - 1 , the reactions were studied without power ultrasound. Results show that size particles sample of 1 - 1 (Fig. 6a) is larger than 1-2 (Fig. 6b). Table 3, shows the average diameter scanning electron micrographs (SEM) of the prepared samples. Results show high power ultrasound irradiation decreased agglomeration, and thus led to decrease particles size. Comparison between samples 1-2 and 1-3 shows a decrease in nanoparticle size. Thus, size particles of sample 1-3 are smaller than 1-2 (Fig. $6 b$ and c). However, a reducing the reaction time led to the decrease of size particles of sample 1-3. Particle sizes and morphology of nanoparticle are depending on temperature. Higher temperature $\left(70^{\circ} \mathrm{C}\right)$ results in an increased solubility, and thus a reduced supersaturation of growth species in the solution, and thus particles size of sample 1-4 is smaller than particles size of sample 1-2 (Fig. 6d, b). Table 3, shows the average diameter of particles shown by scanning electron micrographs (SEM) of the prepared samples. Pay attention to, the best conditions for getting a small sized and less agglomerated nanostructure materials for this hetro-nuclear coordination polymer compound are temperature, reaction time and the power of ultrasonic irradiation $70{ }^{\circ} \mathrm{C}, 60 \mathrm{~min}$ and $60 \mathrm{~W}$, respectively. Also, other condition (temperature $=50^{\circ} \mathrm{C}$, reaction time $=30 \mathrm{~min}$ and power of ultrasonic irradiation $=60 \mathrm{~W}$ ) is good for obtaining small nanoparticle size. 

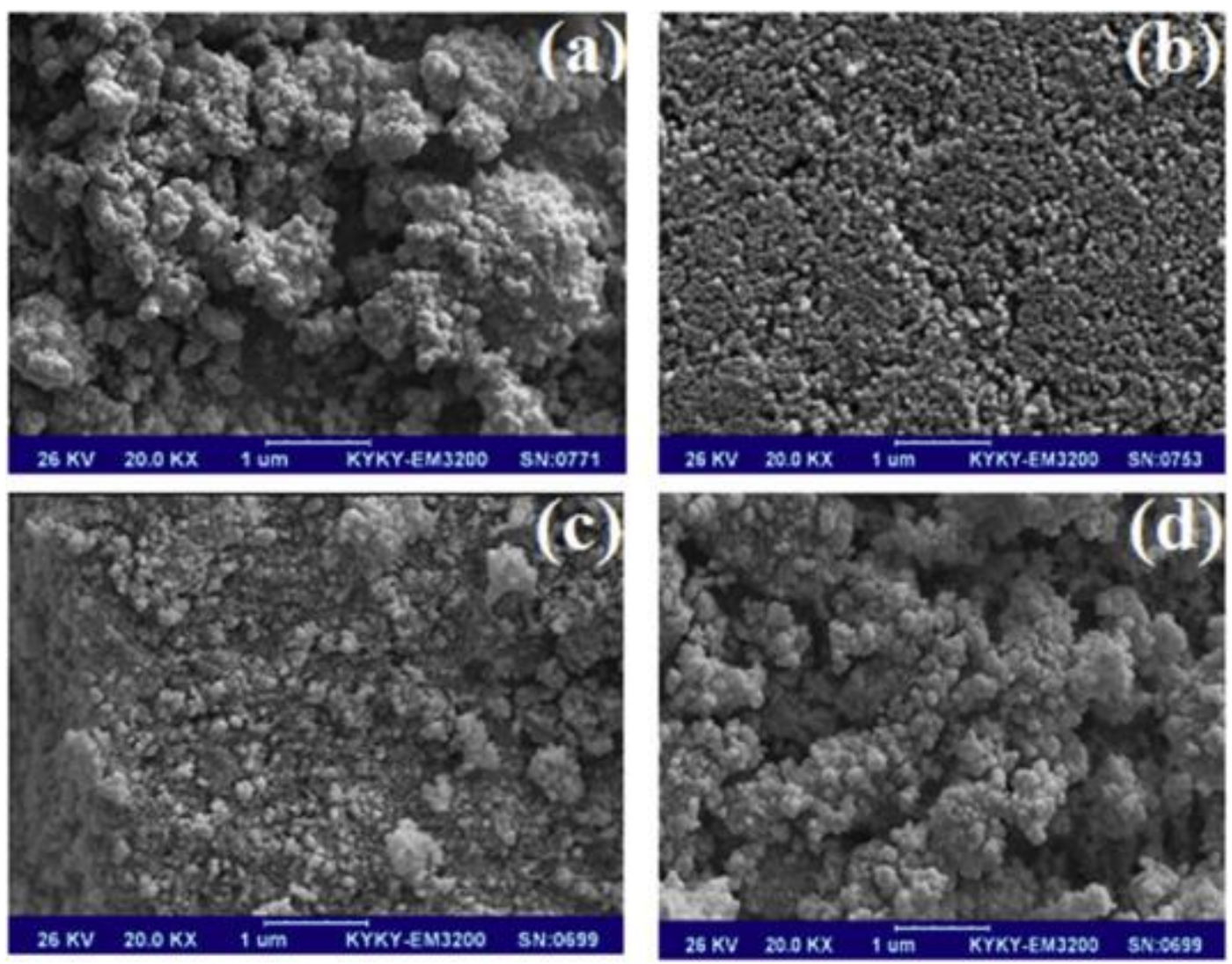

Fig. 6. SEM image of nano particle 1, (a) without sonochemical reaction, (b) by sonochemical reaction with sonochemical of temperature $50 \mathrm{C}$, time of $60 \mathrm{~min}$ and power of $60 \mathrm{~W}$, (c) by sonochemical reaction with sonochemical of temperature 50 $\mathrm{C}$, time of $30 \mathrm{~min}$ and power of $60 \mathrm{~W}$, (d) by sonochemical reaction with sonochemical of temperature $70 \mathrm{C}$, time of $60 \mathrm{~min}$ and power of $60 \mathrm{~W} \cdot \mathrm{P}$. 


\section{Conclusion}

One new $\mathrm{Pb}$ and $\mathrm{K}$ coordination polymer, $\{[\mathrm{Pb} 6(\mathrm{pyc}) 6(\mathrm{~N} 3) 7 \mathrm{~K}] .1 / 2 \mathrm{H} 2 \mathrm{O}\} \mathrm{n}(1)$ (pyc = 2picolinate, $\mathrm{C} 6 \mathrm{H} 4 \mathrm{NO} 2-)$, has been synthesized utilizing a thermal gradient approach and also by sonochemical irradiation. Compound 1 was structurally characterized by single crystal X-ray diffraction. The crystal structure of compound 1 is made up of 1D coordination polymer compound and shows that the coordination number of $\mathrm{Pb}$ ion in compound 1 is seven. Influences of temperature, power ultrasound and reaction time on the morphological properties of $\{[\mathrm{Pb} 6(\mathrm{pyc}) 6(\mathrm{~N} 3) 7 \mathrm{~K}] .1 / 2 \mathrm{H} 2 \mathrm{O}\} \mathrm{n}(1)$, was studied. The results showed that higher temperature of reagents led to decrease in particle size and optimum time for the process was obtained $30 \mathrm{~min}$, increasing in the power of ultrasonic irradiation led to decrease particles size for 1. Also, the best conditions for getting small sized and less agglomerated nanostructure materials are temperature, reaction time and the power of ultrasonic irradiation $70{ }^{\circ} \mathrm{C}, 60 \mathrm{~min}$ and $60 \mathrm{~W}$, respectively.

\section{Acknowledgements}

Support of this investigation by University of Sistan and Baluchestan (USB) and Tarbiat Modares University is gratefully acknowledged.

\section{Appendix A. Supplementary data}

The following is the supplementary data related to this article: Crystallographic data for the structures reported in the paper has been deposited with the Cambridge Crystallographic Data Centre as supplementary publication No: CCDC 1511740 for compounds 1. Copies of the data can be obtained on application to CCDC, 12 Union 
Road, Cambridge CB2 1EZ, UK [Fax: + 44-1223/336033; e-mail:

deposit@ccdc.cam.ac.uk.

1. F.-C. Liu, Y.-F. Zeng, J.-R. Li, X.-H. Bu, H.-J. Zhang, J. Ribas Novel 3-D framework nickel (II) complex with azide, nicotinic acid, and nicotinate (1-) as coligands: hydrothermal synthesis, structure, and magnetic properties Inorg. Chem., 44 (2005), pp. 7298-7300

2. Y. Yang, M.-H. Zeng, X.-H. Zhao, H. Liang Supramolecular networks of hexanuclear cadmium (II): synthesis, crystal structure and emission property Inorganica Chim. Acta, 362 (2009), pp. 3065-3068

3. Y. Bai, W.-L. Shang, D.-B. Dang, J.-D. Sun, H. Gao Synthesis, crystal structure and luminescent properties of one coordination polymer of cadmium (II) with mixed thiocyanate and hexamethylenetetramine ligands Spectrochimica Acta Part A Mol. Biomol. Spectrosc., 72 (2009), pp. 407-411

4. A.V. Davis, K.N. Raymond The big squeeze: guest exchange in an M4L6 supramolecular host J. Am. Chem. Soc., 127 (2005), pp. 7912-7919

5. O.R. Evans, W. Lin Crystal engineering of NLO materials based on metal- organic coordination networks Accounts Chem. Res., 35 (2002), pp. 511-522

6. L. Gao, B. Zhao, G. Li, Z. Shi, S. Feng Inorg. Chem. Commun., 6 (2003), p. 1249

7. S.-W. Jin, W.-Z. Chen Polyhedron, 26 (2007), p. 3074

8. A.M. Goforth, K. Gerth, M.D. Smith, S. Shotwell, U.H.F. Bunz, H.-C. Loye Solid State Sci., 7 (2005), p. 1083

9. P. Hayati, A.R. Rezvani, A. Morsali, P. Retailleau Ultrasound irradiation effect on morphology and size of two new potassium coordination supramolecule compounds Ultrason. Sonochem., 34 (2017), pp. 195-205

10. K. Suslick, M. Fang, T. Hyeon, M. Mdleleni Applications of sonochemistry to materials synthesis Sonochemistry and Sonoluminescence, Springer (1999), pp. 291-320

11. M.J.S. Fard, P. Hayati, A. Firoozadeh, J. Janczak Ultrasonic synthesis of two new zinc (II) bipyridine coordination polymers: new precursors for preparation of zinc (II) oxide nano-particles Ultrason. Sonochem., 35 (2017), pp. 502-513

12. P. Hayati, A.R. Rezvani, A. Morsali, P. Retailleau, S. García-Granda Influences of temperature, power ultrasound and reaction time on the morphological properties of two new mercury (II) coordination supramolecular compounds Ultrason. Sonochem., 34 (2017), pp. 968-977

13. P. Hayati, A. Rezvani, A. Morsali, P. Retailleau, R. Centore Survey of temperature, reaction time and ultrasound irradiation power on sonochemical synthesis of two new nanostructured lead(II) coordination supramolecule compounds Ultrason. Sonochem., 35 (2017), pp. 81-91

14. S.-R. Fan, L.-G. Zhu Syntheses, structures, and characterizations of four new lead(II) 5-sulfosalicylate complexes with both chelating and bridging neutral ligands Inorg. Chem., 46 (2007), pp. 6785-6793

15. M.Y. Masoomi, S. Beheshti, A. Morsali Mechanosynthesis of new azinefunctionalized Zn (II) metal-organic frameworks for improved catalytic performance J. Mater. Chem. A, 2 (2014), pp. 16863-16866

16. J. Yang, J.-F. Ma, Y.-Y. Liu, J.-C. Ma, S.R. Batten Organic-acid effect on the structures of a series of lead(II) complexes Inorg. Chem., 46 (2007), pp. 6542-6555.

17. Zhang, Y.-Y. Qin, Z.-J. Li, Q.-P. Lin, J.-K. Cheng, J. Zhang, Y.-G. Yao Topology analysis and nonlinear-optical-active properties of luminescent metal- organic framework materials based on zinc/lead isophthalates Inorg. Chem., 47 (2008), pp. 8286-8293 
18. M.Y. Masoomi, A. Morsali, P.C. Junk Ultrasound assisted synthesis of a Zn(II) metalorganic framework with nano-plate morphology using non-linear dicarboxylate and linear N-donor ligands RSC Adv., 4 (2014), pp. 47894-47898

19. A. Morsali, M.Y. Masoomi Structures and properties of mercury(II) coordination polymers Coord. Chem. Rev., 253 (2009), pp. 1882-1905

20. A. Morsali, X.-M. Chen A new lead(II) complex of 2, 2'-bipyridine, acetate and thiocyanate ligands: synthesis, characterization and crystal structure of $[\mathrm{Pb}$ (bpy)(NCS)(CH3COO)] n J. Coord. Chem., 57 (2004), pp. 1233-1241

21. Y. Hanifehpour, B. Mirtamizdoust, S.W. Joo Sonochemical synthesis and characterization of the new micro-hexagonal-rod lead (II)-azido coordination compound J. Inorg. Organomet. Polym. Mater., 22 (2012), pp. 916-922

22. H. Sadeghzadeh, A. Morsali New reversible crystal-to-crystal conversion of a mixedligand lead (II) coordination polymer by de-and rehydration Inorg. Chem., 48 (2009), pp. 10871-10873

23. A. Morsali, M. Payeghader, S.S. Monfared, M. Moradi Syntheses and characterization of a new one-dimensional polymer containing ( $\mu$-Thiocyanate)(bpy) lead(II) molecule and new mixed-anion lead(II) complexes: crystal structures of $[\mathrm{Pb}$ (bpy)(SCN)2]n (byp= 2,2'-bipyridine) and $\mathrm{Pb}$ (phen)2(NO3)0.7(ClO4)0.3](ClO4) (phen=1, 10-phenanthroline) J. Coord. Chem., 56 (2003), pp. 761-770

24. E.-Q. Gao, S.-Q. Bai, Y.-F. Yue, Z.-M. Wang, C.-H. Yan New one-dimensional azidobridged manganese(II) coordination polymers exhibiting alternating Ferromagneticantiferromagnetic interactions: structural and magnetic studies Inorg. Chem., 42 (2003), pp. 3642-3649

25. B. Shaabani, B. Mirtamizdoust, D. Viterbo, G. Croce, H. Hammud, P. Hojati, Lalemi, A. Khandar Sonochemical synthesis of a novel nanoscale lead (II) coordination polymer: synthesis, crystal structure, thermal properties, and DFT calculations of $[\mathrm{Pb}(\mathrm{dmp})(\mu-\mathrm{N} 3)(\mu-\mathrm{NO} 3)] \mathrm{n}$ with the novel Pb2( $\mu$-N3)2 ( $\mu$-NO3)2 unit Z. für Anorg. Allg. Chem., 637 (2011), pp. 713-719

26. E.-Q. Gao, Z.-M. Wang, C.-H. Yan From manganese (II)-azido layers to a novel three-dimensional molecular magnet: spin canting and metamagnetism Chem. Commun. (2003), pp. 1748-1749

27. Y. Hanifehpour, B. Mirtamizdoust, A. Morsali, S.W. Joo Sonochemical syntheses of binuclear lead (II)-azido supramolecule with ligand 3, 4, 7, 8-tetramethyl-1, 10phenanthroline as precursor for preparation of lead(II) oxide nanoparticles Ultrason. Sonochem., 23 (2015), pp. 275-281

28. E. Freire, S. Baggio, J.C. Muñoz, R. Baggio Two nickel complexes stabilized by nitrate counter-ions Acta Crystallogr. Sect. C. Cryst. Struct. Commun., 58 (2002), pp. m221-m224

29. C.P. Berlinguette, A. Dragulescu-Andrasi, A. Sieber, J.R. Galán-Mascarós, H.-U. Güdel, C. Achim, K.R. Dunbar A charge-transfer-induced spin transition in the discrete cyanide-bridged complex $\{[\mathrm{Co}(\mathrm{tmphen}) 2] 3[\mathrm{Fe}(\mathrm{CN}) 6] 2\} \mathrm{J}$. Am. Chem. Soc., 126 (2004), pp. 6222-6223

30. A.D. Walmsley, W.R.E. Laird, A.R. Williams A model system to demonstrate the role of cavitational activity in ultrasonic scaling J. Dent. Res., 63 (1984), pp. 1162-1165

31. F. Shahangi Shirazi, K. Akhbari Sonochemical procedures; the main synthetic method for synthesis of coinage metal ion supramolecular polymer nano structures ChemInform, 47 (2016)

32. S.C. Lea, G.J. Price, A.D. Walmsley A study to determine whether cavitation occurs around dental ultrasonic scaling instruments Ultrason. Sonochem, 12 (2005), pp. 233-236

33. F.R. Young Cavitation Imperial College Press, London (1999)

34. B. Felver, D.C. King, S.C. Lea, G.J. Price, A.D. Walmsley Cavitation occurrence around ultrasonic dental scalers Ultrason. Sonochem, 16 (2009), pp. 692-697

35. A.D. Walmsley, S.C. Lea, B. Felver, D.C. King, G.J. Price Mapping cavitation activity around dental ultrasonic tips Clin. Oral Invest., 17 (2013), pp. 1227-1234 
36. G.M. Sheldrichk SHELX-97 University of Göttingen, Germany (1997)

37. O.V. Dolomanov, L.J. Bourhis, R.J. Gildea, J.A.K. Howard, H. Puschmann OLEX2: a complete structure solution, refinement and analysis program J. Appl. Cryst., 42 (2009), pp. 339-341

38. C.F. Macrae, P.R. Edgington, P. McCabe, E. Pidcock, G.P. Shields, R. Taylor, M. Towler, J.v.d Streek Mercury: visualization and analysis of crystal structures J. Appl. Crystallogr., 39 (2006), pp. 453-457

39. A.R. Abbasi, A. Morsali Syntheses and characterization of Agl nano-structures by ultrasonic method: different morphologies under different conditions Ultrason. Sonochem, 17 (2010), pp. 572-578

40. A.R. Abbasi, A. Morsali Formation of silver iodide nanoparticles on silk fiber by means of ultrasonic irradiation Ultrason. Sonochem, 17 (2010), pp. 704-710

41. CnesnN. Eurn, Nnn CW. Worne Crystal habit of synthetic ghalcanthite (copper sulfate pentahydrate) as related to position and orientation in growth solution Am. Mineral., 59 (1974), pp. 1105-1112

42. R.V. Kumar, Y. Diamant, A. Gedanken Sonochemical synthesis and characterization of nanometer-size transition metal oxides from metal acetates Chem. Mater, 12 (2000), pp. 2301-2305

43. Maryam Ghiyasiyan-Arani, Masoud Salavati-Niasari, Sara Naseh Enhanced photodegradation of dye in waste water using iron vanadate nanocomposite; ultrasound-assisted preparation and characterization Ultrason. Sonochem., 39 (2017), pp. 494-503

44. M. Ghiyasiyan-Arani, M. Masjedi-Arani, D. Ghanbari, S. Bagheri, M. Salavati-Niasari Novel chemical synthesis and characterization of copper pyrovanadate nanoparticles and its influence on the flame retardancy of polymeric nanocomposites Sci. Rep., 6 (2016)

45. M. Yousefi, F. Gholamian, D. Ghanbari, M. Salavati-Niasari Polymeric nanocomposite materials: preparation and characterization of star-shaped $\mathrm{PbS}$ nanocrystals and their influence on the thermal stability of acrylonitrile-butadienestyrene (ABS) copolymer Polyhedron, 30 (2011), pp. 1055-1060

46. D. Ghanbari, M. Salavati-Niasari, M. Ghasemi-Kooch A sonochemical method for synthesis of $\mathrm{Fe} 3 \mathrm{O} 4$ nanoparticles and thermal stable PVA-based magnetic nanocomposite J. Ind. Eng. Chem., 20 (2014), pp. 3970-3974

47. D. Ghanbari, M. Salavati-Niasari, S. Karimzadeh, S. Gholamrezaei Hydrothermal synthesis of $\mathrm{Bi} 2 \mathrm{~S} 3$ nanostructures and ABS-based polymeric nanocomposite $\mathrm{J}$. Nanostructures, 4 (2014), pp. 227-232

48. A. Esmaeili-Bafghi-Karimabad, D. Ghanbari, M. Salavati-Niasari, L. NejatiMoghadam, S. Gholamrezaei Photo-catalyst tin dioxide: synthesis and characterization different morphologies of $\mathrm{SnO} 2$ nanostructures and nanocomposites J. Mater. Sci. Mater. Electron., 26 (2015), pp. 6970-6978

49. E. Mirzadeh, K. Akhbari Synthesis of nanomaterials with desirable morphologies from metal-organic frameworks for various applications CrystEngComm, 18 (2016), pp. $7410-7424$

50. K. Akhbari, A. Morsali TI I benzene multicarboxylic acid coordination polymers: structural, thermal and fluorescence studies Inorganica Chim. Acta, 362 (2009), pp. $1692-1700$

51. K. Akhbari, A. Morsali, A.D. Hunter, M. Zeller $[\mathrm{Tl} 2(\mu-\mathrm{Htdp}) 2(\mu-\mathrm{H} 2 \mathrm{O})] n$ ( $\mathrm{H} 2 \mathrm{tdp}=4,4^{\prime}-$ thiodiphenol)-A one-dimensional thallium (I) coordination polymer with a large tetranuclear metallacycle: thermal, emission and structural studies Inorg. Chem. Commun., 10 (2007), pp. 178-182

52. K. Akhbari, A. Morsali Highly polyhapto-aromatic interactions in thallium (I) coordination J. Organomet. Chem., 692 (2007), pp. 5109-5112

53. K. Akhbari, A. Morsali, M. Zeller Unique Ag-C bonds, thermal, fluorescence, structural and solution studies of two-dimensional silver (I) coordination polymer J. Organomet. Chem., 692 (2007), pp. 3788-3795 